\title{
Optimizing First-Line Therapy for Follicular Lymphoma: Is It Time for Chemotherapy-Free Approaches?
}

Presented by Richard I. Fisher, MD

\begin{abstract}
Over the past several decades, tremendous progress has been made in the treatment of follicular lymphoma. The addition of rituximab to chemotherapy led to significant improvements in survival in the 1990s. Current standard of care in advanced-stage, previously untreated follicular lymphoma is rituximab plus chemotherapy, sometimes followed by rituximab maintenance. Now, as more research is conducted in the field of chemotherapy-free treatment, Dr. Richard I. Fisher discussed the importance of carefully constructed phase II or III trials at the NCCN 2019 Annual Congress: Hematologic Malignancies. He maintained that a nonchemotherapy treatment regimen comprising rituximab + lenalidomide can be considered in carefully selected patients, and that it is currently the only chemotherapy-free treatment that should be recommended. J Natl Compr Canc Netw 2019;17(11.5):1414-1416 doi: 10.6004/jnccn.2019.5038
\end{abstract}

In the past several decades, tremendous progress has been made in the treatment of follicular lymphoma (FL), as evidenced by the multiple treatment options available. According to Richard I. Fisher, MD, President, Chief Executive Officer, and Cancer Center Director of Fox Chase Cancer Center, first-line therapy should be limited to one of a few options: watchful waiting, monoclonal antibody (mAb) therapy (rituximab vs obinutuzumab), chemoimmunotherapy (mAb/CHOP [cyclophosphamide/doxorubicin/vincristine/prednisone] vs $\mathrm{mAb}$ /bendamustine), and now, chemotherapy-free treatment with targeted agents. Chemotherapy-free treatment can be considered as a first-line treatment option for FL, but only in selected patients, Dr. Fisher emphasized.

"I would argue that rituximab + lenalidomide $\left[R^{2}\right]$ is the only [nonchemotherapy regimen] that fits that criteria," he said at the NCCN 2019 Annual Congress: Hematologic Malignancies, where he provided an overview of current treatment paradigms for untreated FL.

\section{Conclusions From Past Trials (1970-1990)}

"In the 1970s to the 1990s, overall survival [OS] was not impacted by anything we did," Dr. Fisher said. "Watch and wait" therapy did not impact OS, nor did treatment with alkylating agents, anthracyclines, or combination chemotherapy. Median OS was 7 to 10 years for all patients, and $<20 \%$ were alive at 20 years. According to Dr. Fisher, this led to the consensus that improved OS was not a reasonable endpoint for clinical trials in this particular malignancy.
For decades, the natural history of indolent nonHodgkin's lymphoma remained unchanged. A Stanford study spanning from 1960 to 1991 showed a median survival of 7 to 10 years, with no plateau in the survival curve. ${ }^{1}$

\section{The 1990s: What Changed?}

"Clearly rituximab has had a major impact, and [the addition of] rituximab to chemotherapy changed survival," said Dr. Fisher. This was first seen in a study by Marcus et $\mathrm{al}^{2}$ that compared CVP (cyclophosphamide/ vincristine/prednisone) chemotherapy + rituximab to CVP alone in untreated stage III/IV FL. Median time to treatment failure was 27 months in patients receiving rituximab + CVP and 7 months in the CVP-alone arm $(P<.0001)$.

A German study showed that the addition of rituximab to CHOP (R-CHOP) improved OS over CHOP alone in previously untreated $\mathrm{FL}^{3}{ }^{3}$ and meta-analyses have corroborated these findings. ${ }^{4}$

"You can't find a rituximab/chemotherapy study that isn't better than chemotherapy alone in the treatment of patients with FL," Dr. Fisher noted.

\section{Has Survival Really Improved?}

Evolving therapy, including the incorporation of biologic agents, has led to significant increases in OS and failurefree survival in patients with advanced-stage FL. ${ }^{5}$ At Stanford, median survival from 1987 to 2003 increased to $>19$ years. ${ }^{6}$ 
"Over my career as Chair of the SWOG Lymphoma Committee, there has been a major change in survival with the most recent studies," Dr. Fisher said. "Basically, all of the groups have shown a survival change."

"So think about that: in the last SWOG trial, $90 \%$ of patients were alive and disease-free at 5 to 10 years," he noted. "If that's true, that's a hard-earned victory that we can't give up for our patients."

Then bendamustine came along. In combination with rituximab, it led to superior progression-free survival (PFS) compared with R-CHOP in the frontline treatment of FL, but had no impact on OS. ${ }^{7}$ However, the study did show that rituximab/bendamustine was significantly less toxic. "It was easier to tolerate and has become, de facto, the most commonly used regimen in the United States," he said.

A recently published confirmatory study also showed a PFS benefit with rituximab/bendamustine, with no difference in OS. ${ }^{8}$ "So rituximab/chemotherapy, usually rituximab/bendamustine but sometimes R-CHOP, became the standard of care," Dr. Fisher added.

A study of obinutuzumab/chemotherapy (G-chemo) showed a PFS benefit over rituximab/chemotherapy, but again, with no OS benefit. ${ }^{9}$ According to the authors, G-based therapy can also be considered in the frontline treatment of FL.

\section{Along Came the Biologics: A Cautionary Tale}

The Cancer and Leukemia Group B (CALGB) has led the charge in chemotherapy-free research, first studying combinations of rituximab with various mAbs and subsequently with lenalidomide. ${ }^{10-13}$ All of these studies showed "good response rates and reasonable complete response rates," Dr. Fisher said. However, he also added a cautionary note. "[In the development of chemotherapyfree treatment,] people are very anxious to put together small molecule inhibitors, and that's a really bad idea if you haven't done a very carefully constructed phase I trial," he said.

In a phase I trial that combined $\mathrm{R}^{2}$ with idelalisib, a PI3K inhibitor, patients experienced increased toxicity (pneumonitis, diarrhea/colitis, transaminitis) and severe unexpected toxicity and death. Dr. Fisher said this was a rational trial design that "turned out to be a terrible idea."

In a recently published editorial, ${ }^{14}$ Dr. Bruce Cheson, one of the leaders of the chemotherapy-free movement, discussed this and other recent trials of combinations of agents that were terminated as a result of life-threatening and fatal complications. He called them "speedbumps on the road to a chemotherapy-free world" for patients with lymphoma.

\section{The RELEVANCE Trial}

However, according to Dr. Fisher, a conversation about chemotherapy-free treatment for FL cannot occur without discussing the RELEVANCE trial. ${ }^{13}$ RELEVANCE was the first multicenter, international, open-label, randomized phase III trial of $\mathrm{R}^{2}$ versus rituximab/ chemotherapy followed by rituximab maintenance in previously untreated, advanced FL requiring systemic treatment. Patients in the study were randomized to 1.5 years of $\mathrm{R}^{2}$ followed by a third year of rituximab $(n=513)$ or 6 months of rituximab/chemotherapy followed by a year of rituximab maintenance $(n=517)$. Most patients (72\%) in the rituximab/chemotherapy arm received $\mathrm{R}-\mathrm{CHOP}$.

Dr. Fisher noted that RELEVANCE was set up as a superiority trial, designed to show that $\mathrm{R}^{2}$ is better than rituximab/chemotherapy. However, at 3 years, the trial showed no PFS or OS benefit with $\mathrm{R}^{2}$ compared with rituximab/chemotherapy $(77 \%$ vs $78 \%$, respectively; $P=.48) .{ }^{13}$ "The [survival] curves are basically superimposable," he said. "If you set up a superiority trial and the curves are superimposable, you have a negative study."

Further analysis revealed no evidence of any subset predilection for superiority of $\mathrm{R}^{2}$ in this patient population. Patients in the rituximab/chemotherapy arm experienced more neutropenia and fever; however, overall, treatment discontinuations were similar in both arms: $69 \%$ of patients on the $\mathrm{R}^{2}$ arm and $71 \%$ of those on the rituximab/chemotherapy arm completed treatment. ${ }^{13}$

Final data showed that $\mathrm{R}^{2}$ is not superior to rituximab/chemotherapy, and the 2 treatment regimens demonstrated similar efficacy. Because the study was designed as a superiority trial, it did not meet the desired endpoint. Treatment effects were consistent across subgroups, and continued follow-up for more mature PFS and OS results is ongoing. Based on these data, the study authors concluded that $\mathrm{R}^{2}$, "a novel immunomodulatory approach, is a potential first-line option for patients with FL." 15

"I would not fight that conclusion," Dr. Fisher said, "but I would argue that you need to think about this carefully." He cited the lack of long-term follow-up and the enormous cost differential between $\mathrm{R}^{2}$ and rituximab/chemotherapy (particularly $\mathrm{R}-\mathrm{CHOP}$ ). "We have no idea what the median survival of these patients will be, and we're looking at a group of patients who have a survival not greatly different than age-matched [healthy] people: a little bit worse but not a lot," he noted. "Secondly, we're looking at 3 years of treatment versus potentially $<6$ months of treatment, which is not a trivial difference."

Disclosures: Dr. Fisher has disclosed that he receives consulting fees from Celgene Corporation and PRIME.

Correspondence: Richard I. Fisher, MD, Fox Chase Cancer Center, 333 Cottman Avenue, Philadelphia, PA 19111. Email: richard.fisher@fccc.edu 


\section{References}

1. Horning SJ. Natural history of and therapy for the indolent non-Hodgkin's lymphomas. Semin Oncol 1993;20(5 Suppl 5):75-88.

2. Marcus R, Imrie K, Belch A, et al. CVP chemotherapy plus rituximab compared with CVP as first-line treatment for advanced follicular lymphoma. Blood 2005;105:1417-1423.

3. Hiddemann $W$, Kneba M, Dreyling $M$, et al. Frontline therapy with rituximab added to the combination of cyclophosphamide, doxorubicin, vincristine, and prednisone (CHOP) significantly improves the outcome for patients with advanced-stage follicular lymphoma compared with therapy with $\mathrm{CHOP}$ alone: results of a prospective randomized study of the German Low-Grade Lymphoma Study Group. Blood 2005;106: 3725-3732.

4. Schulz H, Bohlius JF, Trelle S, et al. Immunochemotherapy with rituximab and overall survival in patients with indolent or mantle cell lymphoma: a systematic review and meta-analysis. J Natl Cancer Inst 2007;99:706-714.

5. Liu Q, Fayad L, Cabanillas F, et al. Improvement of overall and failure-free survival in stage IV follicular lymphoma: 25 years of treatment experience at The University of Texas MD Anderson Cancer Center. J Clin Oncol 2006;24:1582-1589.

6. Tan D, Horning SJ, Hoppe RT, et al. Improvements in observed and relative survival in follicular grade 1-2 lymphoma during 4 decades: the Stanford University experience. Blood 2013;122:981-987.

7. Rummel MJ, Niederle N, Maschmeyer G, et al. Bendamustine plus rituximab versus $\mathrm{CHOP}$ plus rituximab as first-line treatment for patients with indolent and mantle-cell lymphomas: an open-label, multicentre, randomised, phase 3 non-inferiority trial. Lancet 2013; 381:1203-1210.

8. Flinn IW, van der Jagt R, Kahl B, et al. First-line treatment of patients with indolent non-Hodgkin lymphoma or mantle-cell lymphoma with bendamustine plus rituximab versus R-CHOP or R-CVP: results of the BRIGHT 5-year follow-up study. J Clin Oncol 2019;37:984-991.

9. Marcus R, Davies A, Ando K, et al. Obinutuzumab for the first-line treatment of follicular lymphoma. N Engl J Med 2017; 377:1331-1344.

10. Czuczman MS, Leonard JP, Jung $S$, et al. Phase II trial of galiximab (anti-CD80 monoclonal antibody) plus rituximab (CALGB 50402): Follicular Lymphoma International Prognostic Index (FLIPI) score is predictive of upfront immunotherapy responsiveness. Ann Oncol 2012. 23:2356-2362

11. Grant BW, Jung SH, Johnson JL, et al. A phase 2 trial of extended induction epratuzumab and rituximab for previously untreated follicular lymphoma: CALGB 50701. Cancer 2013;119:3797-3804.

12. Martin $\mathrm{P}$, Jung $\mathrm{SH}$, Pitcher $\mathrm{B}$, et al. A phase II trial of lenalidomide plus rituximab in previously untreated follicular non-Hodgkin's lymphoma (NHL): CALGB 50803 (Alliance). Ann Oncol 2017; 28:2806-2812.

13. Morschhauser F, Fowler NH, Feugier $P$, et al. Rituximab plus lenalidomide in advanced untreated follicular lymphoma. N Engl J Med 2018; 379:934-947.

14. Cheson BD. Speed bumps on the road to a chemotherapy-free world for lymphoma patients. Blood 2016;128:325-330.

15. Fowler $\mathrm{NH}$, Morschhauser $\mathrm{F}$, Feugier $\mathrm{P}$, et al. Rituximab/Lenalidomide potential frontline therapy for follicular lymphoma. RELEVANCE: phase III randomized study of lenalidomide plus rituximab (R2) versus chemotherapy plus rituximab, followed by rituximab maintenance, in patients with previously untreated follicular lymphoma. Presented at the 2018 ASCO Annual Meeting; June 1-5, 2018; Chicago, Illinois. 